\title{
Methodology to Design for Product Service Supportability
}

\author{
${ }^{1}$ Sarangi Mihir and ${ }^{2}$ Ramgopal Varma Nath \\ 1,2 Department of Mechanical Engineering, Indian Institute of Technology Kharagpur, Kharagpur, India \\ ${ }^{1}$ smihiriitkharagpur@gmail.com
}

\author{
ArticleInfo \\ Journal of Machine and Computing (http://anapub.co.ke/journals/jmc/jmc.html) \\ Doi : https://doi.org/10.53759/7669/jmc202101017 \\ Received 15 March 2021; Revised form 26 April 2021; Accepted 08 June 2021; Available online 05 July 2021. \\ (C2021 The Authors. Published by AnaPub Publications. \\ This is an open access article under the CC BY-NC-ND license. (http://creativecommons.org/licenses/by-nc-nd/4.0/)
}

\begin{abstract}
In today's world, processors must expertly structure sophisticated integrated remedies using modern technologies based on multiple functionalities demands and the rapidly changing perceptions of consumers. Due to this, it is considerably complete for the givers of Product Service Systems (PSSs) to attain all the essential designing requirements. Product designers have to focus on the essential objectives required by PSSs to attain in the whole lifecycle process based on various criteria and approach typically considered in trade-off balancing. Presently, Design-for-X (DfX) approach signifies the most fundamental projection to facilitate production developments based on features and stages of product lifecycle. It is considered that these stages and features support the designing of PSSs, product redesigning and developing engineered products based on $\mathrm{x}$ dimensionality with respect to supportability of services. In this paper, a methodology has been proposed for the generation of novel DfX protocols. As such, an application case in mold industry will be used to represent physical engineered productions, which are developed when services have been integrated or added.
\end{abstract}

Keywords - Product Service Systems (PSSs), Design-for-X (DfX), Design-for-Usability (DfU), Design-for-Quality (DfQ)

\section{INTRODUCTION}

Many researchers have evaluated the transformation of industries. A. Wijayanti, M. Kamalrudin, S. Sidek and K. Hendra Titisari in [1] have focused on the transformation of businesses from traditional to modern with respect to the purchase of physical production and the designing of engineered products. This form of transformation has witnessed wide-range services and products being enabling consumers to attain demanded product functionalities. Various terminologies can be used for this novel business orientation; as for now, literature assumptions direct on servitization, PSSs, Service Domant Logics (SDLs) and Functionality Product (FP). Manufacturing firms that are interested in achieving competitive advantage have focused the novel business orientations widely. Actually, engineering firms have begun their search for integrated services and products to possibly enhance competitive advantage in the market other than offering services such as aftersales service parallel to core product sales.

Integrated remedies transform the process of value creation for consumers from the ownerships of engineered products, physically designed, to consumers' interactions with available artifacts. Some, various activities and knowledge based on the interactions with the remedies' lifecycle can facilitate satisfaction of consumers in the process of value creation. Particularly, the initial stages of the PSSs lifecycle are significant to formulate integrated PSSs that are capable of positively contributing to the value creation process for consumers' process. Different design approaches have to be considered in the process.

Due to this, engineering methods, which are concurrent, e.g. Design-for-X (DfX) have been recommended in research as a fundamental approach for integrating services and engineered products following standard design phases. These approaches have been summarized in Table 1 below. Nonetheless, fewer literature assumptions have extensively focused on effective designing of PSSs product features to achieve PSSs objectives.

Industries today widely on service economy; and for these companies to boost their production performances, PSSs paradigm need presentation to the market. PSSs are featured by the incorporation of services and engineered products bundled into novel remedies to fulfill the required needs of consumers. Nonetheless, engineering firms are not completely aided by these consistent PSSs design approaches, and tools that could allow them to concentrate of perspectives of consumers and firms' internal performance or integrate product and service elements with their lifecycles. Some of the traditional PSSs approaches focused on the going down the drain to fill out this gap.

In addition, more conceptual approaches have been proposed to shift directions; with a sole idea beginning from physical product features and properties, service design integrated in it, without ignoring the lifecycle aspect of the completely integrated remedy. In such competitive and rapidly transforming environment, more concurrent engineering methods e.g. DfX are projected in research, being capable of coping up with various simultaneous challenges of system, process and product design. Mitigating these challenges of traditional sequential engineering, these methods can actually adapt to more physical engineered products in different ways based on PSSs lifecycle and focusing on designers' incompetency in fundamental service and product lifecycle segment. 
Table 1: Fundamental approach for integrating services and engineered products Product Oriented Client Oriented

\begin{tabular}{|c|c|c|c|c|}
\hline \multicolumn{2}{|c|}{ Product Oriented } & & \multicolumn{2}{|c|}{ Client Oriented } \\
\hline $\begin{array}{c}\text { Engineered } \\
\text { Product }\end{array}$ & $\begin{array}{l}\text { Services and } \\
\text { products } \\
\text { - } \quad \text { Maintainabilit } \\
\text { - } \quad \text { Repairability } \\
\text { - } \quad \text { Spare parts } \\
\text { - } \quad \text { Warrants }\end{array}$ & $\begin{array}{cc}\text { Services and } \\
\text { Products } \\
\text { - } & \text { Supplies } \\
\text { - } & \text { upgrades } \\
\text { - } & \text { disposal } \\
\text { - } & \text { Auxiliary } \\
& \text { inputs }\end{array}$ & $\begin{array}{l}\text { Client Activity } \\
\text { Services } \\
\text { - } \text { Planning } \\
\text { - } \text { Designing } \\
\text { - } \text { Specification } \\
\text { - Operations } \\
\text { - } \text { Manufacturing }\end{array}$ & $\begin{array}{cc}\text { Organizational } \\
\text { Supporting Services } \\
\text { - } \quad \text { Consultancy } \\
\text { - Management } \\
\text { - } \text { Partnering } \\
\text { - Consuming }\end{array}$ \\
\hline $\begin{array}{c}\text { Engineered } \\
\text { designing }\end{array}$ & Serviceability design & $\begin{array}{c}\text { Supportability } \\
\text { design }\end{array}$ & Service design & Service design \\
\hline
\end{tabular}

In this research contribution, a methodology producing design protocols and guidelines, developing the Design for Product-Service Supportability (DfPSS) method, focusses on the integration of services and products with lifecycle perspective. With this in mind, this paper has been organized as follows: Section II focusses on a literature survey. Section III provides a critical analysis of the research paper, DfPSS methodology has been evaluated based on protocols and guidelines. Section IV finalizes the paper and provides future research directions.

\section{LITERATURE SURVEY}

H. Honda And T. Murakami in [2] focused on Design-for-Usability (DfU) for user-friendliness of services and products. This was an approach, projected in the early $1980 \mathrm{~s}$, as a strategic approach for designers to incorporate the requirements of interfaces friendly to users. Such kinds of approaches are used in ensuring the safety of consumers in a complex product design process. A scheme designed to be utilized has to be easier to learn and comprehend, relevant (therefore encompassing the relative functions for users) and enjoyable and easier to use.

Y. Yassierli, V. Vinsensius and M. Mohamed in [3] reflect on the aspect of usability with the assurance to users regarding safety use of devices to avoid mistakes during the process of using products; mistakes could cause injuries to users and bring damages to things. Actually, most competent users can incur errors in case the interfaces of users are irrational and ambiguous. This shows the relevance of usability in developing PSSs based on the needs of users. The engagement of users in the design process can enhance the performance of users in the usage of engineered products and enhance the degree of usability. This aids in the definition of certain aspects of system development and specification. In this aspect, Human-Centered Design (HCD) and Activity Centered Design (ACD) have been considered as a fundamental tool for design thinking and which can be utilized to project usability.

M. van der Voort and M. van der Bijl-Brouwer in [4] reviewed three fundamental principles for DfU: initial focus on tasks and users, empirical valuations and iterative designs. Based on usability paradigm and fundamental concepts, the aspect of usability is considered in the balance based on likeability and usability to achieve users' satisfaction with reference to financial and human costs. Utility directly incorporates the functionality of products, which is therefore connected to the aspect of usability. Functionality designs are fundamental in ensuring solution innovations and design quality.

Hyunsuk Han in [5] reviewed the functionality designs as a collection of causal connections between physical parameters according to outward physical device actions. Product functionality was typically attained based on engineering designs, producibility engineering and value engineering methods, which aid designers to diminish product costs after designing. R. Fraccari in [6] review generic guideline-centered approach for functionality.

R. Branaghan in [7] review DfU, which incorporates making devices easier to use. Feedbacks, discoverability and constraints are considered as elements required to the users to enhance their operations in an effective manner using engineered products. Device discoverability is based on the interlinking of knowledge present in the world today. The first one incorporates perceived signifiers and affordance, mapping between various parts to manipulate considering the physical constraints and resultant actions. Physical constraints incorporate conceptual frameworks, logical constraints on behaviors, cultural and semantic constraints between the previous experience and current situations and other futuristic situations.

J. Boissel in [8] presents the idea that designers face practical challenges of comprehending the manner in which things can be designed to make them more comprehensive. To conclude this aspect, we can briefly define usability aspect as the capacity to be utilized by the humans (to a particular dimension of subjective evaluation) and efficiently (to a particular performance level). A number of phases to achieve usability protocols have been done by L. Uden and P. Helo in [9] to put more focus on the designing of interfaces in mobile devices.

P. Basu in [10] has evaluated the Golden Rule of Interface Designing projected to guide the designing and implementations of interfaces for computer machines and their respective applications. In addition, ten different heuristics for the designing of users' interfaces, considering the initially found usability challenges are evaluated. System visibility, matches between real-world and systems, users' freedom and control, standards and consistency, error preventions, recognition instead of recalls, efficiency and flexibility of usage, minimalist and aesthetic designing help the users to recover, diagnose and identify mistakes and errors. 
V. HUBKA in [11] evaluated the Design-for-Quality (DfQ), which focusses on the concept selection and generation; provides functions, many remedies are proposed and most appealing one is selected based on determined method, basically the one reflecting on quality. DfQ is connected to the aspect of functionality more particularly with the evaluation of the how effectively and efficiently the functionality of products can be attained. In addition, quality can be identified as the 'requirement compliance' of the features of machines being capable of enhancing the reliability aspects of products, and application of technology in a progressive manner.

Y. Jo [12] reviewed ISO-9000, which focuses on quality management aspect. This design aspect incorporates services and products, which incorporate traditional design, procedural designing with respect to every product lifecycle and based on Modular Functional Deployment (MFD) and Quality Functional Deployment (QFD). These two elements are a strategic approach for DfQ, which allows the fundamental shift from consumer requirements into more consistent technological specification. In addition, Overall Quality Management (OQM), Six standard and sigma (ISO-9000 are other approaches that are structured for the support for quality management aspects, concentrating of process tasks to attain quality, diminishing defects rating to targeted rates (six standard sigma deviations) or facilitating consumer satisfaction.

Z. Khan in [13] reviewed the Design-for-Assembly (DfA) and the Design-for-Manufacturing (DfM) aspects. These systematic procedures support forms in the optimization of the use of the present manufacturing procedures incorporating the minimization of the number of assembly parts. It has to be noted in the initial stages of the development process that this is parallel to the product concepts and the prototype developments. The aspect of analysis starts conducting DfA to potentially simplify the product structures and to potentially obtain the estimations of costs of various design remedies and assessing the best process and materials.

A. Gebisa and H. Lemu in [14] argue that DfM is effectively implemented on single product parts. These are major DfA assessment approaches: BoothroydDewhurst, Lucas, and Hitachi-AEM approaches. DfM costs evaluation process begins with the analysis of how every part can be manufactured. Design groups can determine alternative production processes, designs, quantification of manufacturing expenses and making essential trade-off decision between various parts consolidations, including the development of manufacturing/material costs.

To wind up, B. G.F and J. Carvalho in [15] argue that DfA and DfM facilitates obtaining of more reliable and simpler engineered products, which are also cost-effective to manufacture and assemble. Out of all the essential factors, there are significant effects on overhead that in most instance form the greatest proportions of the overall product expenses. In addition, different guidelines for DfA and DfM are presently available.

Some of the DfM examples include simplification of designs, designs to minimize labor expenses, elimination of generalized statements on a drawing, dimensions form surface, not from space points. DfA examples include reduction of part type and count, modulirising designs, striving to eliminate the adjustments, designing parts for ease of handling and feeding.

\section{CRITICAL ANALYSIS}

Methodology for Design for Product-Service Supportability (DfPSS) Protocols

This section discussed the methodology for DfPSS generation protocols and guidelines. Fig 2 provides a summary of the methodology. The methodology focusses on formulating design protocols and guidelines to develop the designing of product elements and features supporting and enabling the delivery of quality services. Protocols provide an effective basis that considers generic, lifecycle based data and non-company specific information meant to be followed during repetitive design activities, signifying the featured skills from the company. The methodology and its essential frameworks and tools to manage the generated protocols and rules in consistent repositories have been structured based on various research traditions.

The methodology incorporates six phases that have been grouped into four fundamental sections: Section 1 before begging with content protocols and guideline creation process, preliminary activities have to be completed to collect basic data used in the adoption of the methodology. All DfX techniques that might be incorporated during PSSs designing are gathered and they signify the potential capacities PSSs under designing might represent and achieve with respect to the starting points for the protocols and guidelines definitions. DfX capacity concept is based on the concepts of 'functions' and are the principles for PSSs functions for which they represent the precise guidelines being addressed.

Section 2 is the design process, which is stipulated to start whenever the concept of PSSs is present. Once illustrated in the first phase, the capacities of the products under design has to be attained, and analysis done to form novel suitable rules. Therefore Designing and Technical Requirement (DTR) is illustrated to signify technical and practical projections that have to be embraced by engineers and designers through which capacities might be attained. Thus, novel DTR should be connected to the capacities also signifying the fundamental relationship degrees. With respect to the identified relations between DTR and the capacities, rules are capable of guiding the engineers/signer activities in the system/service/product development, which have to be formalized in text and made present as company's skills.

Section 3 considers the design protocols being created. In this section, the methodology has to guide engineers and designers to concentrate on particular context of the company. To create the relation between PSSs functionalities to be attained and the associated lifecycle variable, which has to be managed, the extended Functionality Transformation Matrix (FTM) approach is applied. A series of these functions, with respect to their structures are used to determine and document the connections among different key factors. These factors include; PSSs features, which are the features of PSSs elements to considered in DTR acts based on the protocols; PSSs lifecycle process, which signifies the PSSs lifecycle activities retrieved from the designing phase to disposal phase; PSSs process variable, which variables that require detection. These belong to any procedures of different phases incorporating the lifecycle of PSSs. 
Lastly, the design protocols are systematically structured with respect to the links identifies in the previous phases. Their major purpose is the capacity-based control of lifecycle variables to effectively managed designing activities to enhance physical PSSs products. Design protocols are actually structured to provide fundamental links for the control of critical variables, which directly include PSSs. Capacities are enhanced by the advent of novel services of the physical products. Section 4 represents the coherence of the various components considered during the process designs and their verifications, which support for engineers and designers in identifying the rightful connections between operative protocols and the high-level protocols. For this purpose, two x-matrices, which have been modified, are applied.

\section{Research Methodology and Case Study}

The case of application in this paper has been done with the main purpose of evaluating and testing the aspect of sustainability of the projected methodology (DfPSS). This research paper focusses on the related merits for firms and their enhanced efficiency, derived from their various applications, in mitigating the issues in PSSs design phases. The case of application was done in two phases. The first one incorporates video sharing with all the members to educate them of the methodology. Therefore, practical workshops have been designed to apply this methodology in the context of engineering firms. Interactive sessions have been led by two experts and incorporated two more professionals with which the firm has long-term relations adding to production tracking workers and a single product designer.

After the actual realization of DfX approaches and level of application in an industrial context, DfPSS method was projected. Thus, a remedy, the mold technical repair history has been identified based on the comprehension of concept design to develop industrial activities. This method have been performed and their design rules and guidelines supporting the designs of novel PSSs obtained. In addition, fundamental responses on the suitability and applicability of the method have been provided.

\section{Case Study}

To further comprehend the methodology, the application case was taken from N-Bazigos-SA, which is a B2B firm in Greece. The Greece-based firm designs and manufactures mold. The designing approaches for the products have been established using CAD and PLM, which are supported by experienced engineering and design divisions. Reviewing servitization process, their real intent is to minimize their ecological implications, waste materials, power use, time-tomarket, failure frequency, design and machining duration; enhance consumer engagement in the designing process and consumer satisfaction; enhance income and competitiveness; access novel marketing segments.

In N-Bazigos-SA, PSSs provisions are in their initial phases of adoption. The services are provided in isolations from the engineered products i.e. the molds without focusing on the interlinked PSSs environment. Nonetheless, the condition of this manufacturing segment dictated at the present that services are indirectly handled. Provided this deficiency of service-based methods in the firm, N-Bazigos-SA is therefore considering novel PSSs activities e.g. the delivery duration of the molds estimated as a service, maintenance histories for consumers, joint consumer-provider proactive management planning for the modification of the molds or opinion extraction provided to consumers as services. This would potentially enhance the aspect of control and monitoring of the molds' lifecycle and minimized downtime of the molds.

The approach was applied in N-Bazigos-SA begin with Section 1, whereby a number of preliminary setting projects comprised of evaluating the as-is of designing processes were considered. The firm does not adopt structured methodologies in designing the molds. However, they embrace basis principles such as the optimization of the molds' lifecycle durations (injection, cooling and ejection of parts). In addition, the design protocols and guidelines, which signify the firm's skills requiring implementation of these methods are note documented or codified and are basically based on the background knowledge of designers.

Moreover, consumers' requirements are significantly linked to the optimization of production, from either a quality or a quantity perspective. In that case, designers are obliged to add on the basic molds and to concentrate of particular aspects of the products' lifecycle in concurrent manner. In most cases, major targets for the design groups are to enhance optimization through more consistent choices of steel adoption, number of pieces being provided with the molds, and to potentially limit downtime. Therefore, whenever steel hardening can be eliminated, firm costs are lower and pricing of consumers is lower; however, this will soon project more maintainability challenges.

To effectively manage this problem, the principles of design for customizability and modularity, incorporating transitional bases and cups, are directly connected to the aspect of maintainability. Contrary to that, utilizing thick plates or considering suitable remedies, molds can become significantly dependable. Nonetheless, it necessitates complex design and maintainability principles, which cannot be ignored. In that case, based on DfPSS methodology, it is fundamental to consider the designing of already present molds to enhance the aspect of functionality (mostly based on the maintainability perspective) and to comprehend the components that are likely to transform.

A novel remedy, capable of meeting the needs of the firm was noted: technical repair history of the molds. Therefore, products requiring redesigning, with respect to consumer operation in the plastic firm, were identified: 1 liter-seal-lid molds and 2 cavity mold. Table 2 below shows the major challenges of the components: 1 liter-seal-lid molds and 2 cavity mold. 
Table 2: Major challenges of 1 liter-seal-lid molds and 2 cavity mold

Element

$\begin{array}{ll}\begin{array}{l}\text { Core and } \\ \text { cavities }\end{array} & \begin{array}{l}\text { Dual halves for the molds, which form } \\ \text { plasmatic geometries of products }\end{array}\end{array}$

Cooling Insertation, which bears injection holes

bushes from which plastics flow and bears cooling circuits

$\begin{array}{ll}\begin{array}{l}\text { Hotrunner } \\ \text { systems }\end{array} & \text { Given by specific distributions and } \\ & \begin{array}{l}\text { suppliers, it can distribute plastic } \\ \text { materials to many cavities }\end{array}\end{array}$

Stripper Components, which shift in relative

rings motions to core eject plastic parts from different molds

\section{Issues}

They typically bear centering components, i.e. two segments that have to be aligned with high degree of precision

Holes are damaged by the flows of materials. They are also designed for manufacturability insertation and therefore can be replaced

The tips of the nozzles including different points of contact with precise fittings are damages using the flows of materials

Precise fitting are needed and because of their physical wear, they have to be replaced

Generation of Protocols and Guidelines

With respect to the analysis of the present design method of the firm, based on the methodology process in Section 1 , the identified the remedy to be presented, DfPSS, protocols and guidelines were formulated based on the phases defined in Section $2 \& 4$. Other than the purpose to guide the formation of products consistent to the needs of consumers, the design protocols and guidelines are fundamental to govern reworks because they are considered to provide exact data regarding the design process of products. Fig 2 provides a summary of the finds based on the application of Section 2, which starts in the first phase, projecting on the capacities' definitions, 5 essential capacities to structure the remedy were noted. The remedy was based on the physical product features required for PSSs provision: traceability, identity coding, disassembly/assembly operation, inspectability, maintainability and modularity.

\begin{tabular}{|c|c|c|c|c|c|c|c|c|c|c|c|c|c|}
\hline & 4 & 4 & & & OTR6_Mounting features for moving and handling & & & & & & & & 4 \\
\hline 5 & & 2 & 3 & 2 & OTR5_Component engraving & & & & & & & & \\
\hline 5 & & 2 & 3 & 2 & OTRA_Unique BOM coding & & & & & & 3 & & \\
\hline & 4 & 3 & & 2 & OTR3_Connectivity of hydraulic and automatic connection & & & & & 3 & & 3 & \\
\hline 4 & & & 4 & 4 & OTR2_Oesign \& manufacture standard components (families) & & 4 & & 4 & & & & \\
\hline 3 & & & 4 & 4 & OTR1_Use standard components (from suppliers) & 4 & & 4 & & & & & \\
\hline 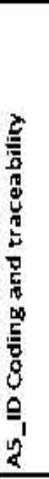 & 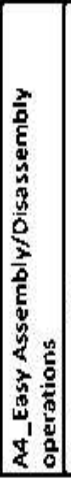 & 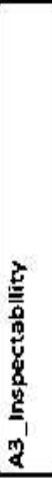 & 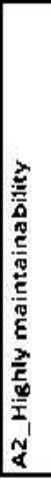 & $\begin{array}{l}\frac{z}{5} \\
\frac{5}{5} \\
\frac{5}{5} \\
\frac{8}{2} \\
\frac{1}{8} \\
\end{array}$ & GUIDELINES & 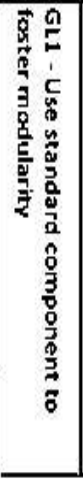 & 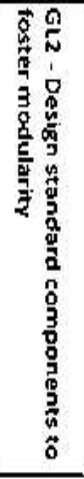 & 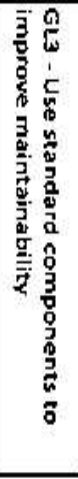 & 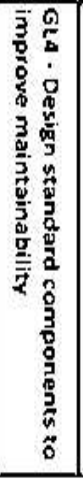 & 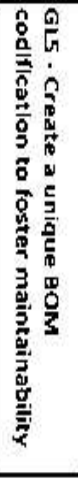 & 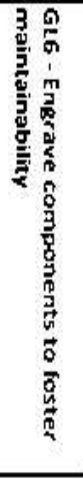 & 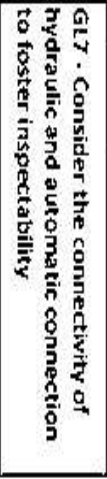 & 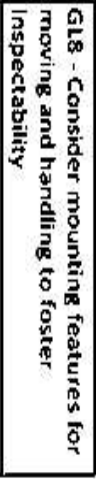 \\
\hline
\end{tabular}

Fig 1: FTM X segment matrix from remedies to protocols

In the second phase, six different DTRs were illustrated based on the past capacities; utilize standardized elements from the suppliers, manufacturing and designing standardized elements for product groups, connectivity of automatic and hydraulic connections, exceptional bom codings, element engraving, mounting characters for handling and moving. Therefore, the connections' grade between every DTR and the capacities was noted. Resultantly, 14 protocols were retrieved since the connections with the weight higher or equal to three were termed fundamental and therefore translated and analyzed as operational protocols of designers.

Section 3, précised in Fig 1 was done to formulate detailed protocols. In the $3^{\text {rd }}$ phase, PSSs characteristics were noted. They were projected to enhance the capacities, noted in the previous phases, of some fundamental elements, e.g. hot-runner, governing elements, cooling bushes, socket, centering components, water manifolds, hole monitoring, and cone centering. Therefore, the group comprehended repetitively to focus on the connections between PSSs and the 
protocols. In the $4^{\text {th }}$ phase, the attention of designers shifted to the lifecycle procedure and step documentation; particularly the seven stages noted ie concepts \& designing, manufacturing, assembling, validations, usage, maintainability and disposals. The definitions of the connections between PSSs and lifecycle processes helped engineers to comprehend the existing value of PSSs in the various stages of the remedy lifecycle. Again, the resultant connections fostered the formulation of the design protocols to be embraced by the product designers.

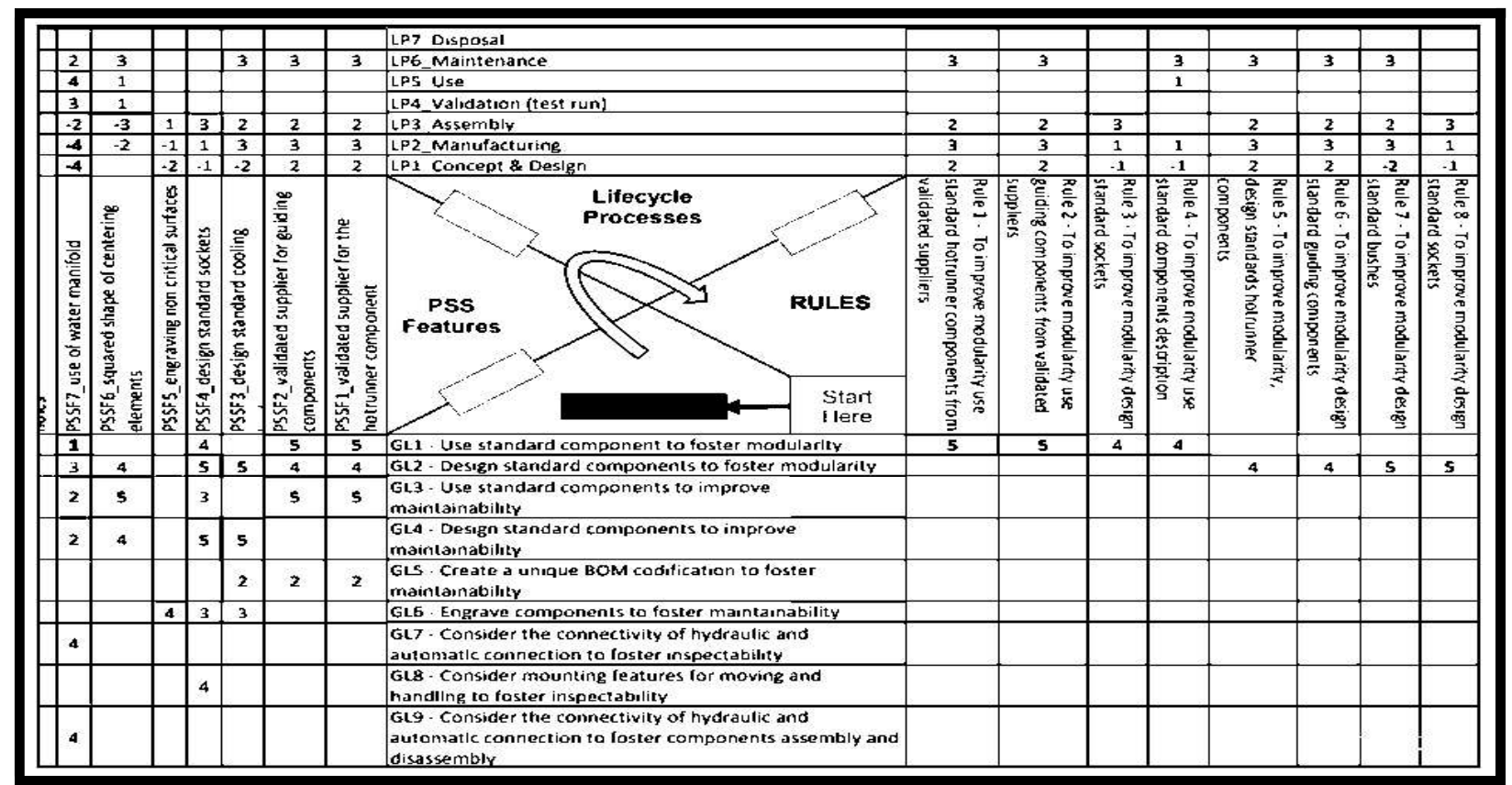

Fig 2: FTM X segment matrix for lifecycle phases

Lastly, the $5^{\text {th }}$ phase required the group to check and determine the coherence between the various data formulated with respect to DfPSS following the analysis of the x-matrix. No significant demerits were evident. Only a single protocol (GL5), not being connected to any provided PSSF was not explicated in particular protocols. In addition, the protocols connected to PSSF6 and PSSF7 were featured by considerably negative weights at the start of the PSSs lifecycle, hence amounting to the requirement of more significant efforts for the firm; designers have to consider this following critical trade-off thinking. Nonetheless, various merits would be realized following this accomplishment.

For instance, to attain A3, DTR6, mounting the characteristics for handling and shifting; and DTR3, linkage of automatic and hydraulic connections, were noted. Particularly, the connection of A3 and DTR3 was significantly in GL7 (based on the connections of automatic and hydraulic linkage for fostering the aspect of inspectablity). To categorically work on this, PSSF7 was considered. This characteristic necessitated fundamental efforts at the start of the PSSs lifecycle (design \& concepts, and manufacturing \& assemblies); however, making validation tests to run easier hence providing huge enhancements in the usage and maintainability stages.

\section{CONCLUSION AND FUTURE DIRECTIONS}

Several results have been recorded using the application case in this paper. The major evidence is that the recommended is capable of mitigating the issues in product engineering, fostering service and product characteristics incorporation in determine PSSs designs. Particularly, based on the methodology, fourteen novel protocols and guidelineconnected protocols were checked and obtained. Responses gathered during the methodological applications in the industrial cases study would be viewed as additional results: the variation between protocols and guidelines could be afformed using the way they are encrypted (such as considering the application of passive tense for protocols and $\mathrm{x}$ matrices visualization might be developed to automatically illustrate potential results. Their major concerns with the methodologies concerning the efforts required to apply it is compared standardized processes.

N-Bazigos-SA is one of the Small and Medium Enterprise (SME). In that case, product designers are allowed to design according to their preference, always minding the manufaturability of molds but without requiring continuous designing an innovative thing. Molds, B2B industrial products have to satisfy the requirements and preference of users, its innovations might be considered applicable and strategic from a service perspective, confirming the significance of DfPSS concept. Actually, the methodological adoption could necessitate designed to invest more time to achieve various concepts introduced (even when it amounted to easy-to-follow) and to formulated the obtained skills in repositories of tools. In SMEs, it is challenging to transform routines and to operate using novel tools. As such, professional designers would emphasize that they do not require applying the recommend approach since they are familiar with the design protocols. 
The methodology appeared to be significantly fundamental to capture comprehensive design stages but also representing structured ways to manage it. However, this also diminishes, to a particular degree, a sense of relaxation purposed to obtain novel ideas. With respect to N-Bazigos-SA workers, the methodology can enhance in a fundamental way if PSSs design phases are applied to engineering firms at a global level. Larger firms are normally involved in progressive innovations of their remedies, following strict requirements and embracing stronger resource structures capable of exploiting this process in a comprehensive manner. In addition, with its implementation, problem-solving procedure would be speeded and simplified up also alongside the spaces, in various industrial plans scattered in various places, and the timeframe, incorporating various designers' generation and to foster collaborations among firms' networks and divisions.

This paper has evaluated the manner in which engineering firms can be supported during service feature integration in the designing of products of PSSs. To receive empirical responses in an industrial context, the methodology projecting DfPSS protocols and guidelines should be adopted as evident in the case of application. This has therefore been done in the SME to produce molds for B2B markets, willing to undergo servitization. Therefore, among the present products that belong to the firm's portfolio, the remedy to be formulated and released to the consumers as PSSs has been detected: injection of molds for the plastic firm's maintainability with respect to technological history of the repair.

Based on this application case, physical engineered products were developed and service characteristics integrated in them. Actually, the methodology confirmed to be firmly engineered and projected to develop novel DfX-based methods for the development of PSSs and at easing the issue solving process, normal for the design phases, and balancing tradeoffs with different capacities meant to be satisfied. The application case was done to allow engineers and designers to effectively apply the methodology. With reference to the application case, the projected methodology would amount to more advantages to even larger firms, but necessitating maintainability in their respective designs. In smaller firms, where competent designers train their inexperienced designers, the valuable skill remains tactical.

Lastly, with respect to the above discussion, more tests could be considered in the future (for global firms) to assess the design methodology, not just for the sake of SMEs. Novel sector-particular DfPSS protocols and guidelines were acquired. As such, the provisions of tools, utilized as repositories for specific protocols and generic guidelines can ease the activities of designers in the design stage. This skillset can be connected through the application of tags, in the design activities or PSSs capacities. This skillsets, consistently filtered can be reapplied for future design activities. With respect to this, the tools, already applied in the case study, will be given and developed by engineers.

\section{References}

[1]. A. Wijayanti, M. Kamalrudin, S. Sidek and K. Hendra Titisari, "A business transformation model to enhance the sustainability of small-sized family businesses", Problems and Perspectives in Management, vol. 19, no. 1, pp. 185-197, 2021. Available: 10.21511/ppm.19(1).2021.16.

[2]. H. Honda And T. Murakami, "Usability Design Support Uniformly Applicable through Upstream to Downstream Design Phases", The Proceedings of Design \& Systems Conference, vol. 201929, no. 0, p. 1412, 2019. Available: 10.1299/jsmedsd.2019.29.1412.

[3]. Y. Yassierli, V. Vinsensius and M. Mohamed, "The Importance of Usability Aspect in M-Commerce Application for Satisfaction and Continuance Intention", Makara Journal of Technology, vol. 22, no. 3, p. 149, 2019. Available: 10.7454/mst.v22i3.3655.

[4]. M. van der Voort and M. van der Bijl-Brouwer, "Designing for Dynamic Usability: Development of a Design Method that Supports Designing Products for Dynamic Use Situations", Design Principles and Practices: An International Journal-Annual Review, vol. 2, no. 1, pp. 149-158, 2008. Available: 10.18848/1833-1874/cgp/v02i01/38119.

[5]. Hyunsuk Han, "A Research on Functionality of Design - Focusing on Examples of Architecture, Transportation, and Product Designs -", $A$ Journal of Brand Design Association of Korea, vol. 13, no. 2, pp. 125-134, 2015. Available: 10.18852/bdak.2015.13.2.125.

[6]. R. Fraccari, "Lipid Bilayer Coated Nanopipettes as Generic Nanopore Sensors with Enhanced Functionality", Biophysical Journal, vol. 108, no. 2, p. 326a, 2015. Available: 10.1016/j.bpj.2014.11.1775.

[7]. R. Branaghan, "Book Review: Usability Assessment: How to Measure the Usability of Products, Services, and Systems", Ergonomics in Design: The Quarterly of Human Factors Applications, vol. 25, no. 3, pp. 29-30, 2017. Available: 10.1177/1064804617713175a.

[8]. J. Boissel, "Controlled clinical trials: Today's challenges for statisticians and designers", Controlled Clinical Trials, vol. 1, no. 4, pp. 333-337, 1981. Available: 10.1016/0197-2456(81)90037-4.

[9]. L. Uden and P. Helo, "Designing mobile interfaces using activity theory", International Journal of Mobile Communications, vol. 6, no. 5, p. 616, 2008. Available: 10.1504/ijmc.2008.019325.

[10]. P. Basu, "Effect of interface roughness on excitonic linewidth in a quantum well: Golden-rule and self-consistent-Born-approximation calculations", Physical Review B, vol. 44, no. 16, pp. 8798-8801, 1991. Available: 10.1103/physrevb.44.8798.

[11]. V. HUBKA, "Design for Quality and Design Methodology", Journal of Engineering Design, vol. 3, no. 1, pp. 5-15, 1992. Available: $10.1080 / 09544829208914744$.

[12]. Y. Jo, "ISO9000 and the civil engineering industry in a borderless world.", Doboku Gakkai Ronbunshu, no. 522, pp. 1-17, 1995. Available: 10.2208/jscej.1995.522_1.

[13]. Z. Khan, "Design for assembly", Assembly Automation, vol. 28, no. 3, pp. 200-206, 2008. Available: 10.1108/01445150810889961.

[14]. A. Gebisa and H. Lemu, "Design for manufacturing to design for Additive Manufacturing: Analysis of implications for design optimality and product sustainability", Procedia Manufacturing, vol. 13, pp. 724-731, 2017. Available: 10.1016/j.promfg.2017.09.120

[15]. B. G.F and J. Carvalho, "Design for Manufacturing and Assembly methodology applied to aircrafts design and manufacturing", IFAC Proceedings Volumes, vol. 46, no. 7, pp. 116-121, 2013. Available: 10.3182/20130522-3-br-4036.00044. 